\title{
Protective Effect of Ethanol Extract of Legundi (Vitex trifolia L.) Leaves against Staphylococcus aureus in Drosophila Infection Model
}

\author{
Yuni Sukarsih 1(D), Rudi Arfiansyah 1(D), Tri Puspita Roska ${ }^{1}{ }^{\mathbb{D}}$, Mufidah Murdifin ${ }^{1}{ }^{\mathbb{D}}$, Syaharuddin \\ Kasim $^{1}$ (D), Firzan Nainu ${ }^{1, *}$ (D) \\ 1 Faculty of Pharmacy, Universitas Hasanuddin, Makassar 90245, Indonesia \\ * Correspondence: firzannainu@ unhas.ac.id;
}

Scopus Author ID 57120069200

Received: 25.01.2021; Revised: 21.02.2021; Accepted: 25.02.2021; Published: 2.03.2021

\begin{abstract}
Research to discover certain medicinal plants' antibacterial activity against Staphylococcus aureus was mostly performed in vitro. The purpose of this research was to investigate the antibacterial activity of ethanol extract of legundi leaves (EELL) against S. aureus using model organism Drosophila melanogaster. The extract was prepared by the maceration method using $70 \%$ ethanol. The antibacterial activities of EELL were determined by using fly survival assay and bacterial colony-forming assay. Fly survival assay was conducted to investigate the extracts' ability to enhance the survival of $D$. melanogaster (host) upon S. aureus infection. The results demonstrated that both EELL were able to increase the survival rate of the S. aureus-infected Drosophila. Furthermore, a colony-forming assay was carried out to determine the growth of bacteria in the host body that has been considered an important pathogenic factor for the host. The result found that the number of bacteria recovered from the EELL-treated infected flies was significantly lower than the ones obtained from the infected flies without any treatments. Overall, EELL protects the $S$. aureus-infected hosts, suggesting the potential antibacterial effect of EELL against $S$. aureus.
\end{abstract}

Keywords: Vitex trifolia; antibacterial; gram-positive; Staphylococcus aureus; fruit fly.

(C) 2021 by the authors. This article is an open-access article distributed under the terms and conditions of the Creative Commons Attribution (CC BY) license (https://creativecommons.org/licenses/by/4.0/).

\section{Introduction}

Infectious diseases are considered major health problems that continue to rise worldwide [1, 2], including in Indonesia [3]. Infectious diseases can be caused by four major groups of microorganisms; bacteria, fungi, viruses, and parasites [4]. One example of bacteria that can cause infection is Staphylococcus aureus [5]. Almost everyone has experienced $S$. aureus infections during life, from mild skin infections to life-threatening nosocomial infections [5, 6]. Treatment of diseases caused by $S$. aureus infection was previously performed using $\beta$-lactam group antibiotics, such as penicillin, cephalosporin, and their derivatives [7]. However, this pathogen rapidly becomes resistant to many antibiotics [8, 9], creating massive problems worldwide. Increased resistance of $S$. aureus to different types of antibiotics eventually remarks the urgent need for new antibiotics with novel mechanisms of action [10, 11]. Many efforts have been put at stake to discover antimicrobial drugs with such specific modalities to achieve the purpose.

Antibiotics can be obtained from different sources, either isolated from natural resources or by chemical synthesizing from available materials [12-14]. An example of natural 
sources prospective for antimicrobial activity is legundi (Vitex trifolia L.) [15]. Extract of legundi (V. trifolia L.) leaves shown to have antibacterial properties against $S$. aureus $[16,17]$. However, the result was obtained under the in vitro condition (agar diffusion method). In the field of drug discovery, results obtained in the in vitro assay sometimes do not reflect the actual condition that may happen when using animal models or even humans [18, 19]. For example, some antimicrobial compounds undergo pharmacokinetic metabolism before exerting their antibacterial effect [20]. In such cases, the original compounds may produce less antibacterial inhibitory profiles in the in vitro setting. Therefore, we believed that it is necessary to carry out further testing to determine the prospective antibacterial effect of Vitex trifolia leaves extract in vivo (using animal test).

Currently, the fruit fly (Drosophila melanogaster) becomes one of the alternatives in vivo platforms that are being developed internationally as a model organism to examine physiological and pathological conditions of eukaryotic organ systems, sometimes recapitulating human conditions [21-23]. Drosophila melanogaster has many advantages, such as similar genetic makeup to humans (about 65\%) [22], can be infected with human pathogenic bacteria and amenable for antimicrobial treatments [24-29], faster reproduction, lower maintenance, and testing costs, and do not require a code of ethics use of experimental animals in the study [22, 30, 31]. In addition, drugs used to treat diseases in humans may have similar therapeutic effects on D. melanogaster, such as celecoxib, doxorubicin, doxycycline, L-Dopa, phenytoin, and some antibiotics such as tetracycline, rifampicin, and linezolid [32-34].

The application of the insect model system in the anti-infective drug discovery was exemplified by Hamamoto et al. (2015) in the discovery of Lysosin E antibiotics with new mechanisms of action [35]. The notion that insects, particularly D. melanogaster, can be used as a model organism(s) at the initial stage of antimicrobials drug discovery has been supported by published literature [24-27, 36, 37]. With the support of information availability and various types of mutants, D. melanogaster is highly potential to be used as a model organism in the preliminary testing of antimicrobial candidates [31] and the investigation of contributing factors in the pathogenesis of emerging infectious diseases, including Coronavirus Disease (COVID)-19 [38]. This research was conducted to determine the protective effect of ethanol extract of legundi leaves (EELL) against $S$. aureus using model organism D. melanogaster. The results obtained here will provide information on the prospective antibacterial effect of EELL in the in vivo condition.

\section{Materials and Methods}

\subsection{Bacterial strains, fly stocks, and preparation of fly infection model.}

Staphylococcus aureus ATCC 29213 strain was used in all infection experiments conducted in this study. Preparation of $S$. aureus infection model using adult male $w^{1118} D$. melanogaster at the age of 4-7 days old was carried in this study, using previously established procedure [26, 27]. Briefly, S. aureus was cultured in Nutrient Broth (NB) medium for $1 \times 24$ hours at $37^{\circ} \mathrm{C}$, collected, and washed several times with PBS followed by resuspension in PBS before use. The in vivo infection model was prepared by needle pricking method, piercing the fly thorax with a special needle that has been previously dipped into the $S$. aureus suspension. Before the injection process, flies were anesthetized with $\mathrm{CO}_{2}$. After pricking, flies were transferred into vials containing standard fly food. Subsequently, they were observed for several minutes until all flies recovered from anesthesia ( $\pm 15-20$ minutes). At this stage, dead 
flies were excluded for further process. After recovery, flies were finally transferred to new vials corresponds to their intended treatments (in the presence or absence of antibiotics or EELL at certain concentrations), followed by subsequent experiments. All flies were maintained in standard laboratory conditions (12 hours light and 12 hours dark cycle, standard cornmeal-agar food, $25^{\circ} \mathrm{C}$ ).

\subsection{Plant extract preparation.}

Legundi (V. trifolia L.) leaves were collected from some of the areas in Makassar, South Sulawesi, Indonesia. Samples were processed through wet sortation and subsequently dried in an oven at $40^{\circ} \mathrm{C}$. The legundi leaves were then extracted by maceration with ethanol $70 \%$ for $1 \times 24$ hours followed by 2 times re-maceration processes. The solvent of the filtrate was evaporated with a rotary evaporator to obtain the suitable thickened ethanol extract of legundi leaves (EELL). Finally, the extract was dissolved in distilled water before incorporation into fly food until intended concentrations of EELL (1\%; 5\%; and 25\%) were achieved.

\subsection{Survival assay.}

Five groups of $S$. aureus-infected Drosophila and one group of mock-infected Drosophila (healthy control) were prepared. Thirty adult males of The $w^{1118}$ flies were assigned randomly into each group. Of five groups of $S$. aureus-infected Drosophila, one group was assigned as the negative control group (fed only with standard fly food), and one group was assigned as the positive control group (fed with fly food containing tetracycline $200 \mu \mathrm{g} / \mathrm{g}$ ). The remaining three groups of $S$. aureus-infected Drosophila were allocated as treatment groups (fed with fly food containing either $1 \%, 5 \%$, or $25 \%$ of EELL). All fly groups were incubated at $29^{\circ} \mathrm{C}$ and monitored daily for survivorship by observing the number of dead flies every day until no live flies were observed in the negative control group (Fig. 1). Results were recorded and processed using GraphPad Prism ${ }^{\circledR} 8$, and data is prepared as a Kaplan-Meier curve and statistically analyzed with the Log-Rank Test.

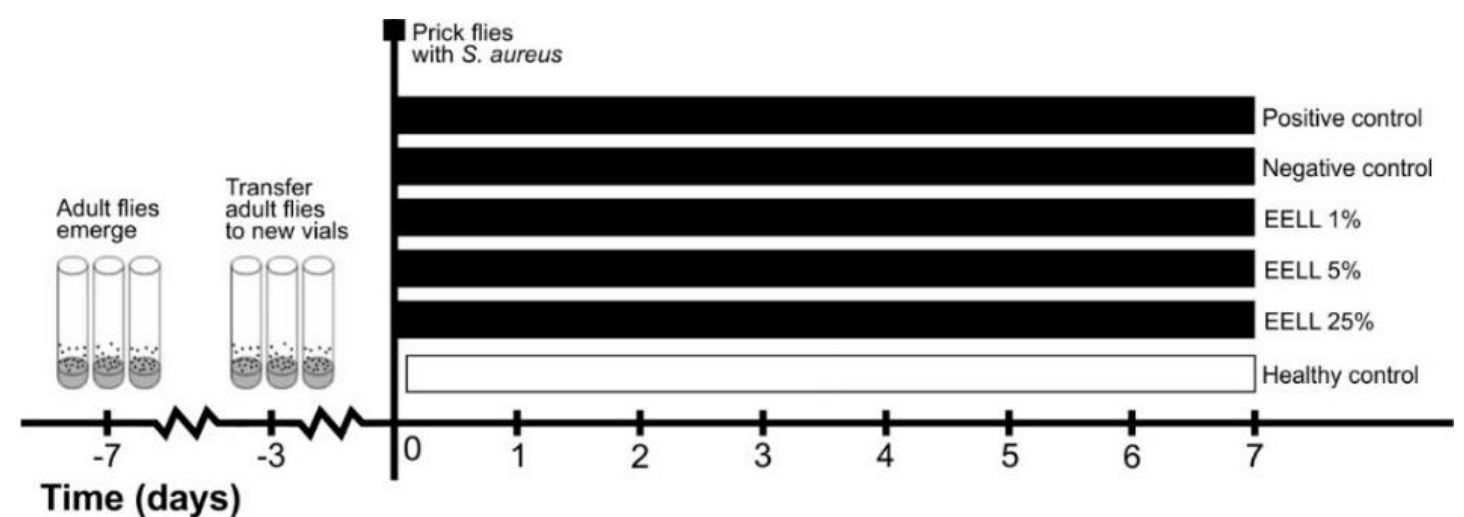

Figure 1. Timeline for fly preparation and observation of survival rate in the infection experiment. Adult males of the ${ }^{\text {w11 }}$ D. melanogaster at 4-7 days old age were assigned into six groups. All flies, except those in the healthy control group, were subjected to a pricking procedure with $S$. aureus inoculum and then transferred into vials containing intended treatments. Flies in the positive control group were treated with tetracycline, and the ones in the negative control group received no additional treatment other than standard fly food. The remaining three groups of S. aureus-infected flies were separately subjected to EELL treatment at a concentration of either $1 \%$, $5 \%$, or $25 \%$. Survival rates were observed daily after bacterial injection. The observation was ended on day 7 post pricking. EELL: ethanol extract of legundi leaves. 


\subsection{Colony-forming assay.}

Three groups of S. aureus-infected Drosophila and one group of mock-infected Drosophila (healthy control) were prepared as described in section 2.3. All groups (with 10 flies in each group) were maintained at $29^{\circ} \mathrm{C}$, then observed daily until 72 hours post-infection. Five live flies of each group were then separately transferred into a Treff tube then manually crushed using a micropestle. During that process, 100 $\mu$ PBS (Phosphate Buffer Saline) was added to the tube to obtain a homogeneous concentrate liquid. The homogenate was then subjected to centrifugation at 5,000 rpm for 3 minutes until the supernatant was separated from the precipitate. To reduce the bacterial cells' density, a serial dilution of the obtained supernatant was carried out by a 10-fold serial dilution (10 $\mu 1$ into $90 \mu \mathrm{PBS}$ and up to 10-3 dilution was obtained). After that, $50 \mu \mathrm{L}$ from each dilution was dispersed in a Petri dish containing Vogel-Johnson Agar (VJA) medium with 1\% potassium tellurite. The number of viable $S$. aureus colonies grown after overnight incubation at $37^{\circ} \mathrm{C}$ was counted and expressed as $\mathrm{cfu} / \mathrm{ml}$. The results were processed and analyzed using the GraphPad Prism ${ }^{\circledR} 8$. All data were reported as mean \pm S.D, and statistical analysis by one-way ANOVA was performed. A simplified timeline for this procedure can be seen in Fig. 2.

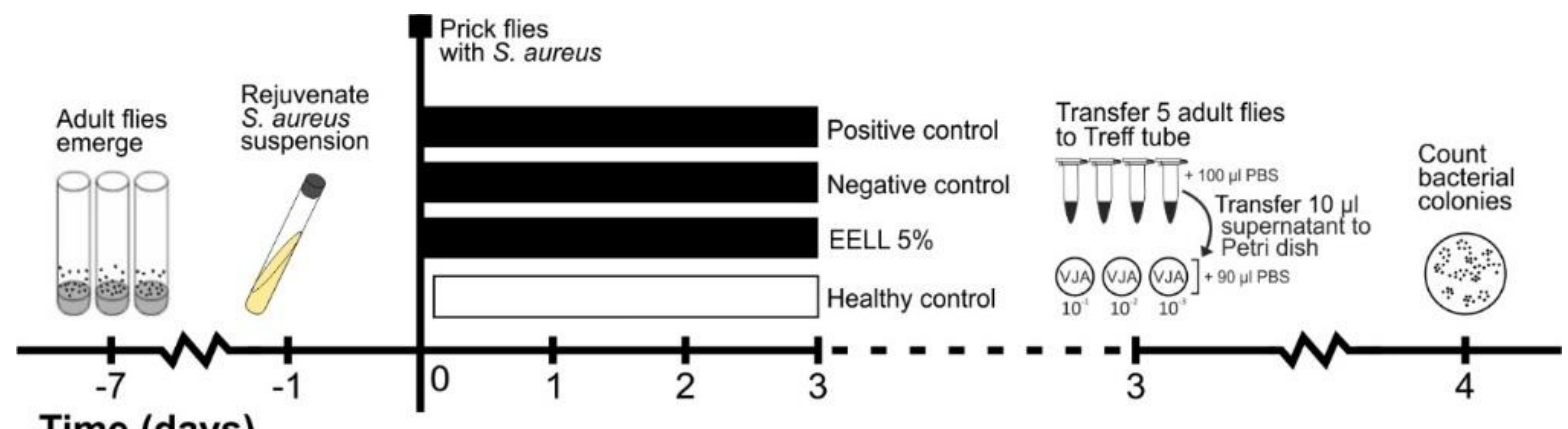

Time (days)

Figure 2. Timeline for colony-forming assay. Adult males of ${ }^{w 1118}$ D. melanogaster at 4-7 days old of age were assigned into four groups. All flies, except those in the healthy control group, were subjected to a pricking procedure with $S$. aureus inoculum and then transferred into vials containing intended treatments. Flies in the positive control group were treated with tetracycline, and the ones in the negative control group received no additional treatment other than standard fly food. The EELL-treated group was given EELL treatment at a concentration of 5\%. Survival rates were observed daily after bacterial injection until 72 hours post-infection. EELL: ethanol extract of legundi leaves.

\section{Results and Discussion}

\subsection{Enhancement of S. aureus-infected flies survival in the presence of EELL.}

Based on the results of survival assay analysis (Fig. 3), it can be seen that around 60\% of flies in the negative control group were succumbed on the third days after being pricked with S. aureus. In comparison, $90 \%$ population of the healthy control group survived until the end of the study. Such discrepancy of survival between these two groups indicated the negative effect of $S$. aureus on the fly lifespan, possibly due to infection, as suggested in our previous studies [24, 27]. Furthermore, around $80 \%$ of flies in the positive control group (which were treated with tetracycline $200 \mu \mathrm{g} / \mathrm{g}$ contained in their fly food) could survive at three days post pricking with $S$. aureus. As shown in Fig. 3, the survival rate of tetracycline-treated flies was positively improved, indicating that tetracycline administration can yield a protective effect on $S$. aureus-infected D. melanogaster. In addition to that, results of the survival assay shown by 
all control groups used in this study were similar to our previous publications [24, 27]. This was important to reassure that our infection procedure was performed properly manner.

The survival rates of EELL-treated flies at a concentration of $5 \%$ and $25 \%$ were positively improved compared to the S. aureus-infected Drosophila in the negative control group. However, the flies treated with $1 \%$ of EELL demonstrated no improvement in the survival rate upon $S$. aureus infection, suggesting that at $1 \%$ concentration, the amount of antibacterial compound(s) in the EELL might be insufficient to overcome bacterial infections. Nevertheless, it is important to note that the survival rate of flies shown in Fig. 3 signifies the importance of EELL improving the likelihood of S. aureus-infected D. melanogaster to survive during the course of infection. In addition to that, since $5 \%$ and $25 \%$ of EELL yielded a similar survival profile, we believed that $5 \%$ of EELL would be sufficient to be used in the next experiment.

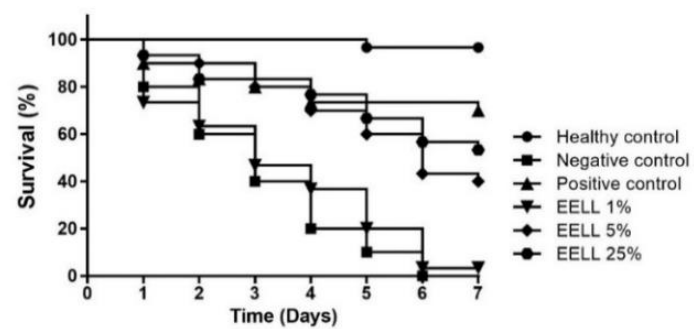

Figure 3. Survival rate of $S$. aureus-infected flies in the presence or absence of EELL. Adult male $w^{1118}$ flies at 4-7 days after eclosion were infected with $S$. aureus by pricking followed by subsequent treatment with fly food containing either tetracycline (positive control) or EELL at a concentration of $1 \%, 5 \%$ or $25 \%$. Healthy flies were given normal fly food (healthy control) and $S$. aureus-infected flies in the absence of any treatments (negative control) were also included in the assay. All groups were subjected to survival assay. EELL: ethanol extract of legundi leaves.

\subsection{Inhibition of S. aureus growth in infected flies upon EELL treatment.}

We have shown that improvement of fly survival, as seen in Fig. 3 can be achieved by reducing bacterial propagation in the infected flies irrespective of bacterial species [24-27]. To examine whether this notion is true in this particular study, we performed a colony-forming assay. In this assay, we calculated the number of $S$. aureus (that can be recovered from the infected flies) after serial dilution and incubation on VJA. As can be in Fig. 4, the number of $S$. aureus colonies recovered from the flies in the positive control group was significantly lower than the ones obtained from the negative control group, further supporting the notion that increased fly survival (Fig. 3) was linearly correlated to bacterial propagation (Fig. 4).

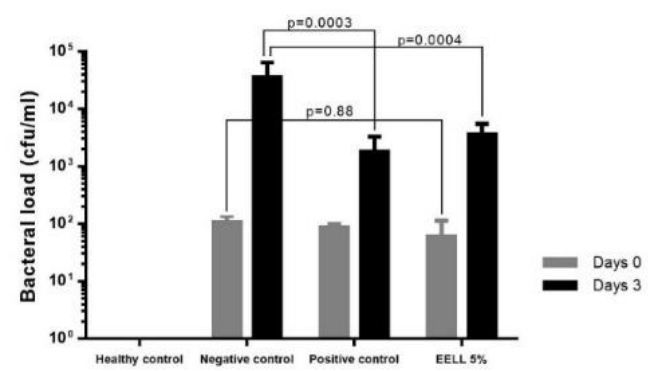

Figure 4. Reduction of bacterial propagation in $S$. aureus-infected flies in the presence EELL. Adult male $w^{1118}$ flies at 4-7 days after eclosion were infected with $S$. aureus by pricking followed by subsequent treatment with fly food containing either tetracycline (positive control) or EELL at a concentration of 5\%. Healthy flies given normal fly food (healthy control) and $S$. aureus-infected flies in the absence of any treatments (negative control) were also included in the assay. All groups were subjected to colony-forming assay. EELL: ethanol extract of legundi leaves. 
To examine the effect of EELL on bacterial propagation, we carried out a similar colony-forming assay experiment. Our result (Fig. 4) showed that the treatment of 5\% EELL to $S$. aureus-infected flies could reduce the number of recovered bacteria, reported as CFU/mL, demonstrating the significance of EELL to negatively affect the growth of $S$. aureus in Drosophila model of infection in vivo.

\section{Conclusions}

Ethanol extract of legundi (V. trifolia L.) leaves (EELL) yielded in vivo antibacterial activity against $S$. aureus in the Drosophila model of infection. While the results presented here could not provide detailed explanations on the anti-staphylococcal mechanism of action of EELL, we could at least demonstrate for the first time the antibacterial effect of legundi ( $V$. trifolia L.) leaves against $S$. aureus in an in vivo insect platform. Future studies to elucidate the prospective antibacterial candidates before pharmacological and toxicological assays in higher eukaryotes, including non-human primates, are highly encouraged.

\section{Funding}

This research received no external funding.

\section{Acknowledgments}

We want to offer our gratitude to Prof. Yoshinobu Nakanishi (Kanazawa University, Japan) for his kind support in providing Drosophila lines used in this study.

\section{Conflicts of Interest}

The authors declare no conflict of interest.

\section{References}

1. Fauci, A.S. Infectious diseases: considerations for the 21 st century. Clin Infect Dis 2001, 32, 675-685, https://doi.org/10.1086/319235.

2. Bloom, D.E.; Cadarette, D. Infectious Disease Threats in the Twenty-First Century: Strengthening the Global Response. Front Immunol 2019, 10, 549-549, https://doi.org/10.3389/fimmu.2019.00549.

3. Nelwan, E.J. The Threat of Emerging and Re-emerging Infections in Indonesia. Acta Med Indones 2019, 51, 195-196,

4. Verhoef, J.; van Kessel, K.; Snippe, H. Immune Response in Human Pathology: Infections Caused by Bacteria, Viruses, Fungi, and Parasites. Nijkamp and Parnham's Principles of Immunopharmacology 2019, 165-178, https://doi.org/10.1007/978-3-030-10811-3_10.

5. Tong, S.Y.C.; Davis, J.S.; Eichenberger, E.; Holland, T.L.; Fowler, V.G.Jr. Staphylococcus aureus infections: epidemiology, pathophysiology, clinical manifestations, and management. Clin Microbiol Rev 2015, 28, 603-661, https://doi.org/10.1128/CMR.00134-14.

6. Turner, N.A.; Sharma-Kuinkel, B.K.; Maskarinec, S.A.; Eichenberger, E.M.; Shah, P.P.; Carugati, M.; Holland, T.L.; Fowler, V.G. Methicillin-resistant Staphylococcus aureus: an overview of basic and clinical research. Nature Reviews Microbiology 2019, 17, 203-218, https://doi.org/10.1038/s41579-018-0147-4.

7. Hutchings, M.I.; Truman, A.W.; Wilkinson, B. Antibiotics: past, present and future. Current Opinion in Microbiology 2019, 51, 72-80, https://doi.org/10.1016/j.mib.2019.10.008.

8. Vestergaard, M.; Frees, D.; Ingmer, H. Antibiotic Resistance and the MRSA Problem. Microbiology Spectrum 2019, 7, https://doi.org/10.1128/microbiolspec.GPP3-0057-2018.

9. Abushaheen, M.A.; Muzaheed; Fatani, A.J.; Alosaimi, M.; Mansy, W.; George, M.; Acharya, S.; Rathod, S.; Divakar, D.D.; Jhugroo, C.; Vellappally, S.; Khan, A.A.; Shaik, J.; Jhugroo, P. Antimicrobial resistance, mechanisms and its clinical significance. Disease-a-Month 2020, 66, https://doi.org/10.1016/j.disamonth.2020.100971.

10. Chambers, H.F.; Deleo, F.R. Waves of resistance: Staphylococcus aureus in the antibiotic era. Nat Rev Microbiol 2009, 7, 629-641, https://doi.org/10.1038/nrmicro2200. 
11. Duval, R.E.; Grare, M.; Demoré, B. Fight Against Antimicrobial Resistance: We Always Need New Antibacterials but for Right Bacteria. Molecules 2019, 24, https://doi.org/10.3390/molecules24173152.

12. Lewis, K. Platforms for antibiotic discovery. Nat Rev Drug Discov 2013, 12, 371-387, https://doi.org/10.1038/nrd3975.

13. Wright, G.D. Something old, something new: revisiting natural products in antibiotic drug discovery. Can J Microbiol 2014, 60, 147-154, https://doi.org/10.1139/cjm-2014-0063.

14. Álvarez-Martínez, F.J.; Barrajón-Catalán, E.; Micol, V. Tackling Antibiotic Resistance with Compounds of Natural Origin: A Comprehensive Review. Biomedicines 2020, 8 , https://doi.org/10.3390/biomedicines8100405.

15. Kannathasan, K.; Senthilkumar, A.; Venkatesalu, V. In vitro antibacterial potential of some Vitex species against human pathogenic bacteria. Asian Pac J Trop Med 2011, 4, 645-648, https://doi.org/10.1016/s19957645(11)60164-8.

16. Hossain, M.M.; Paul, N.; Sohrab, M.H.; Rahman, E.; Rashid, M.A. Antibacterial activity of Vitex trifolia. Fitoterapia 2001, 72, 695-697, https://doi.org/10.1016/S0367-326X(01)00304-5.

17. Natheer, S.E.; Sekar, C.; Amutharaj, P.; Rahman, M.S.A.; Khan, K.F. Evaluation of antibacterial activity of Morinda citrifolia, Vitex trifolia and Chromolaena odorata. African Journal of Pharmacy and Pharmacology 2012, 6, 783-788.

18. Edmondson, R.; Broglie, J.J.; Adcock, A.F.; Yang, L. Three-dimensional cell culture systems and their applications in drug discovery and cell-based biosensors. Assay Drug Dev Technol 2014, 12, 207-218, https://doi.org/10.1089/adt.2014.573.

19. Theuretzbacher, U.; Outterson, K.; Engel, A.; Karlén, A. The global preclinical antibacterial pipeline. Nature Reviews Microbiology 2020, 18, 275-285, https://doi.org/10.1038/s41579-019-0288-0.

20. Levison, M.E.; Levison, J.H. Pharmacokinetics and pharmacodynamics of antibacterial agents. Infect Dis Clin North Am 2009, 23, 791-815, https://doi.org/10.1016/j.idc.2009.06.008.

21. Asri, R.M.; Salim, E.; Nainu, F.; Hori, A.; Kuraishi, T. Sterile induction of innate immunity in Drosophila melanogaster. Frontiers in Bioscience (Landmark Edition) 2019, 24, 1390-1400, http://dx.doi.org/10.2741/4786.

22. Pandey, U.B.; Nichols, C.D. Human disease models in Drosophila melanogaster and the role of the fly in therapeutic drug discovery. Pharmacol Rev 2011, 63, 411-436, https://doi.org/10.1124/pr.110.003293.

23. Nainu, F.; Shiratsuchi, A.; Nakanishi, Y. Induction of apoptosis and subsequent phagocytosis of virusinfected cells as an antiviral mechanism. Front Immunol 2017, 8, 1220-1220, https://doi.org/10.3389/fimmu.2017.01220.

24. Nainu, F.; Asri, R.M.; Arsyad, A.; Manggau, M.A.; Amir, M.N. In vivo antibacterial activity of green algae Ulva reticulata against Staphylococcus aureus in Drosophila model of infection. Pharmacognosy Journal 2018, 10, 993-997, https://doi.org/10.5530/pj.2018.5.169.

25. Nainu, F.; Asri, R. M.; Djide, M. N.; Ahsan, M.; Arfiansyah, R.; Sartini, S.; Alam, G. Protective effect of green algae Ulva reticulata against Pseudomonas aeruginosa in Drosophila infection model. HAYATI Journal of Biosciences 2019, 26, 163-171.

26. Nainu, F.; Djide, M. N.; Subehan, S.; Sartini, S.; Roska, T. P.; Salim, E.; Kuraishi, T. Protective Signatures of Roselle (Hibiscus sabdariffa L.) Calyx Fractions against Staphylococcus aureus in Drosophila Infection Model. HAYATI Journal of Biosciences 2020, 27, 306-313.

27. Ahsan, M.; Gonsales, A. V.; Sartini, S.; Wahyudin, E.; Nainu, F. In vivo anti-staphylococcal activity of roselle (Hibiscus sabdariffa L.) calyx extract in Drosophila model of infection. Journal of Herbmed Pharmacology 2019, 8, 41-46, https://doi.org/10.15171/jhp.2019.07.

28. Chamilos, G.; Samonis, G.; Kontoyiannis, D.P. Drosophila melanogaster as a model host for the study of microbial pathogenicity and the discovery of novel antimicrobial compounds. Curr Pharm Des 2011, 17, 1246-1253, https://doi.org/10.2174/138161211795703744.

29. Tzelepis, I.; Kapsetaki, S.-E.; Panayidou, S.; Apidianakis, Y. Drosophila melanogaster: a first step and a stepping-stone to anti-infectives. Curr Opin Pharmacol 2013, 13, 763-768, https://doi.org/10.1016/j.coph.2013.08.003.

30. Ugur, B.; Chen, K.; Bellen, H. J. Drosophila tools and assays for the study of human diseases. Dis Model Mech 2016, 9, 235-244, https://doi.org/10.1242/dmm.023762.

31. Nainu, F.; Nakanishi, Y.; Shiratsuchi, A. Fruit fly as a model organism in the study of human diseases and drug discovery. Journal of Center for Medical Education Sapporo Medical University 2019, 21-32,

32. Fernández-Hernández, I.; Scheenaard, E.; Pollarolo, G.; Gonzalez, C. The translational relevance of Drosophila in drug discovery. EMBO Rep 2016, 17, 471-472, https://doi.org/10.15252/embr.201642080.

33. Ben-Ami, R.; Watson, C.C.; Lewis, R.E.; Albert, N.D.; Arias, C.A.; Raad, II; Kontoyiannis, D.P. Drosophila melanogaster as a model to explore the effects of methicillin-resistant Staphylococcus aureus strain type on virulence and response to linezolid treatment. Microb Pathog 2013, 55, 16-20, https://doi.org/10.1016/j.micpath.2012.11.012.

34. Apidianakis, Y.; Rahme, L.G. Drosophila melanogaster as a model host for studying Pseudomonas aeruginosa infection. Nature Protocols 2009, 4, 1285-1294, https://doi.org/10.1038/nprot.2009.124. 
35. Hamamoto, H.; Urai, M.; Ishii, K.; Yasukawa, J.; Paudel, A.; Murai, M.; Kaji, T.; Kuranaga, T.; Hamase, K.; Katsu, T.; Su, J.; Adachi, T.; Uchida, R.; Tomoda, H.; Yamada, M.; Souma, M.; Kurihara, H.; Inoue, M.; Sekimizu, K. Lysocin E is a new antibiotic that targets menaquinone in the bacterial membrane. Nat Chem Biol 2015, 11, 127-133, https://doi.org/10.1038/nchembio.1710.

36. Ekowati, H.; Arai, J.; Putri, A. S. D.; Nainu, F.; Shiratsuchi, A.; Nakanishi, Y. Protective effects of Phaseolus vulgaris lectin against viral infection in Drosophila. Drug Discoveries and Therapeutics 2017, 11, 329-335, https://doi.org/10.5582/ddt.2017.01071.

37. Panayidou, S.; Ioannidou, E.; Apidianakis, Y. Human pathogenic bacteria, fungi, and viruses in Drosophila: disease modeling, lessons, and shortcomings. Virulence 2014, 5, 253-269, https://doi.org/10.4161/viru.27524.

38. Nainu, F.; Rahmatika, D.; Emran, T.B.; Harapan, H. Potential application of Drosophila melanogaster as a model organism in COVID-19-related research. Frontiers in Pharmacology 2020, 11, https://doi.org/10.3389/fphar.2020.588561. 